International Journal on Cybernetics \& Informatics (IJCI) Vol. 5, No. 2, April 2016

\title{
EMG ANALYSIS AND CONTROL OF ARTIFICIAL ARM
}

\author{
Anjali Raghavan ${ }^{1} \&$ Prof. Sunny Joseph ${ }^{2}$ \\ ${ }^{1}$ Department of Electronics and Communication, Mar Athanasius College Of \\ Engineering, \\ A.P.J Abdul Kalam Technological University,Kerala, India \\ ${ }^{2}$ Associate Prof, Department of Electronics and Communication, \\ Mar Athanasius College Of Engineering, Kothamangalam, Kerala, India
}

\begin{abstract}
Robotic hand for prosthetic applications is a unique structure intended to be driven by electro myographic (EMG) signals captured from human body. The main characteristic of this robotic hand is its actuation system, which is based on the behaviour of EMG signals. The direct relation between signal and actuation system lends itself well to interpreting the EMG signals from the muscles into effective task execution, with the goal of helping the user to achieve a good approximation of the full capabilities associated with the human hand, without compromising strength, dexterity, appearance, or weight; which are common issues associated with prosthetic hands. EMG signal capturing capability was added to control the DC motors. The emg signal was then filtered and scaled using MAT lab to a value representing the amplitude of the EMG signal, which was then used to control the direction of the motors. The controller is a simple feed forward system in the project but provides the appropriate framework to integrate more elaborate control schemes and EMG signal conditioning. The goal is to create a direct relation between the EMG signals.
\end{abstract}

\section{KEYWORDS}

Robotic hand, prosthetic, EMG, DC motor, MAT lab

\section{INTRODUCTION}

There have been many different approaches taken in the development of an effective prosthetic hand. These varying strategies often find themselves focusing on one of the following categories: implementing a new actuator type, developing a more effective kinematic structure, integrating effective compliance, generating effective control strategies, and interpreting/conditioning input signals. Advances in these areas have resulted in robotic hands that perform many tasks with a high similarity to that of the human hand, such as the DLR hand, I-Limb hand, Shadow hand, and Fluid hand to name a few. However, a prosthetic hand that is nimble, quick, strong, lightweight, quiet, and efficient has yet to be achieved. The primary reason for the current state of prosthetic hands has been the complexity associated with the human hand as a result of its multiple bones and joints. This is further compounded by the fact that the human hand as a functioning unit does not just embody the palm and its digits but also the wrist, forearm muscles, nervous system, and the body's energy generation system. As a result, the entire prosthetic hand actuation structure (inputs, power, strength, kinematics, etc.) must fit in a significantly reduced volume compared to DOI: $10.5121 /$ ijci.2016.5234 
the human hand that it is replacing. To address some of the challenges described above, this project implements a unique perspective of the muscles signals in the human forearm and proposes a novel design and parallel actuation structure that complements this perspective. The goal is to create a direct relation between the forearm's EMG signals and the actuation system, in order to help the user achieve a good approximation of the full capabilities associated with the human hand in a compact design.

\subsection{Motivation of the work}

Cerebrovascular accident (CVA) also known as stroke is being the major cause of long term disabilities. The only way to aid stroke patient is restoring loss through rehabilitation training, which can be enhance using rehabilitation device. As referred, the training can be enhanced using exoskeleton systems by guiding patients in relearning motions on correct trajectories, or by giving them force support to perform certain motions. However, mathematical representation of human lower extremities is required in order to build the device. From the past studies, it is clear that EMG system provide changes of neuromuscular system while the muscles experiencing fatigue. Thus here relationship between EMG signal and human movement is studied.

\section{ELECTROMYOGRAM}

Electromyography (EMG) is a technique for evaluating and recording the electrical activity produced by skeletal muscles.[2] An electromyograph detects the electrical potential generated by muscle cells when these cells are electrically or neurologically activated. The signals can be analyzed to detect medical abnormalities, activation level, and recruitment order or to analyze the biomechanics of human or animal movement. Understanding EMG implies understanding muscles and the way they generate bioelectric signals. It also implies how specific mechanisms influence the signals as well as how the signals reflect certain mechanisms and phenomena and allow their identification and description. The EMG signal is a representation of the electric potential field generated by the depolarization of the outer muscle membrane. Its detection involves the use of intramuscular or surface electrodes that are placed at a certain distance from the sources. The surface EMG signal is an effective and important system input for the control of powered prosthesis. This control approach, referred to as myoelectric control, has found widespread use for individuals with amputations or congenitally weak limbs. The filtered form of obtained EMG signal can be used for these purposes.

Understanding EMG Signals implies the understanding of muscles and the way they generate bioelectric signals. A basic description of the physiological system that generates the EMG signal is presented here.

\subsection{Prosthetic Hand Design}

The design is dimensionally consistent with that of an average male human hand and possesses the same degrees of freedom.[3] The anthropomorphic aspect of the hand is intended to enhance the amputee's acceptance and usability. The DIP and PIP joints of the finger and the IP and MCP joints of the thumb are coupled. This is achieved by connecting a single actuator to both the PIP joint (bevel gears) and DIP joint (pulley connection on metacarpal phalange). The movement associated with a region is achieved by two DC motors. The DC motors actuating the coupled 
DIP/PIP joints of the finger and IP/MCP joints of the thumb are embedded in the proximal phalange of the finger and the metacarpal phalange of the thumb. The DC motor in the metacarpal phalange of the finger actuates the horizontal degree of freedom of the MCP joint. The DC motor at the base of the thumb actuates the CMC joint to obtain an approximation of the abduction/adduction motion. The second degree of freedom of the finger's MCP joint (abduction/adduction) is only subject to compliance without actuation. The second degree of freedom in the thumb's CMC joint (flexion/extension) is actuated by another region actuation structure. The actuation structure corresponding to other region for the finger includes a light cable that passes over two restraining shafts in the MCP joint of the finger, coils in the proximal phalange, and embeds in the middle phalange. The string is kept in light tension by a tension unit at the back of the hand while that region actuation structure is active. When other region actuation is required the shape memory alloy actuates a spring loaded cam which in turn pinches the string between itself and a roller beneath it. As the shape memory alloy continues to actuate, the cam introduces the additional force required for other region tasks. At task completion the electric signal causing the shape memory alloy to heat up is stopped and the DC motors and cam spring extend the shape memory alloy back to its original state. The thumb's region 2 actuation structure is similar to that of the finger's region 2 actuation structure. However, unlike the finger, this structure actuates the degree of freedom at the CMC joint that is not actuated by the DC motor. This is based on the observation that this degree of freedom is more apposing of the fingers during tasks that would require additional force. The design shown in this section has been manufactured using a rapid prototyping machine.

\subsection{Human-Machine Interface: Processing of The EMG Signal}

The user can control the grasping task adopting a consolidated technique, the processing of the EMG signals[2]. Two EMG signals generated by two antagonist muscles of the forearm devoted to the wrist flexion-extension movement (the extensor carpi radialis and the flexor carpi radialis) are acquired and processed by two small boards (each 0.8 " by 1.5 " in dimension), in the first board, the signal is rectified and filtered (pre-processing phase); in the second one, the preprocessed EMG-signal is used to control the actuators of the RTR II hand (low level control). The function of the two boards is to interpret the user's intention and to send the appropriate commands to the actuators. Two small boards are used to preprocess the EMG signal and to control the actuators of the RTR II hand.

\subsection{EMG Pre-Processing}

The first board receives as inputs two EMG signal measured by 2 Delsys DE2.3 differential Ag electrodes, designed with a built-in gain of $1000 \mathrm{~V} / \mathrm{V}$ and a built-in pass band filter from $20 \pm 5 \mathrm{~Hz}$ to $450 \pm 50 \mathrm{~Hz}$. These signals are then rectified (by a full-bridge precision rectifier with adjustable gain) and low-pass filtered through a Butterworth filter (2nd order, pass-band edge frequency: 8 $\mathrm{Hz}$, pass-band attenuation: $0.5 \mathrm{~dB}, \mathrm{fp}=13.56 \mathrm{~Hz}$ ), whose transfer function is:

$$
\mathrm{T}(\mathrm{s})=T 0 \frac{A}{B s 2+C s+D} .
$$

Where $\mathrm{T} 0=1, \mathrm{~A}=7.23 \mathrm{E} 3, \mathrm{~B}=1, \mathrm{C}=1.2 \mathrm{E} 2, \mathrm{D}=7.2 \mathrm{E} 3$. 
The two rectified signals are then compared in order to detect the direction of movement (i.e. extension=open, flexion=close; SGN signal); moreover, the signal is also compared with an adjustable threshold with a regenerative comparator, in order to detect if there is a movement or not (EDG signal).

\subsection{Low Level Control of the Hand}

The second board receives 3 signals from the first one: activation (EDG), direction (SGN) and amplitude of the movement (AMP).[1] The first signal (EDG) is used to understand if there is a movement or not; the 2nd (SGN) to 2232 choose between opening and closing, and the 3rd (AMP) to control the speed of the movement in a proportional way. The second board is composed of 2 blocks: the control block, which is composed by a PIC16F870 microcontroller, and the driver block, which contains 2 drivers capable of driving 2 motors up to $18 \mathrm{~W}$ each. The control algorithm (in this first prototype) is a simple proportional open loop control. It remains in the stand-by state (low power consumption) until there is a positive edge of the EDG signal. The controller reads the SGN signal (SGN0), and starts to sample the AMP signal (obtained by the rectified difference of the 2 rectified EMG signals). After twait (twait $=300 \mathrm{msec}$ in this first version of the controlling algorithm) the controller reads once again the SGN signal (SGN1). If SGN1 6= SGN0, it means that the user is changing the control from one motor to the other. Instead, if SGN1 = SGN0, the controller starts to drive the motor of the hand by using the Pulse Width Modulation (PWM) technique. The duty cycle is calculated from the average of the previous 64 samples of the EMG signal. The direction of movement is determined by the SGN signal. During twait the microcontroller samples the EMG signal, and averages the last 128 samples. If this average is greater than a pre-determined threshold for more than $70 \%$ of the time, it means that the user wants to move the hand at low speed. The next version of the control algorithm, currently under development, will exploit the force and position sensors, in order to control locally some grasping functions.

\section{BLOCK DIAGRAM AND FLOWCHART}

\subsection{Block Diagram}

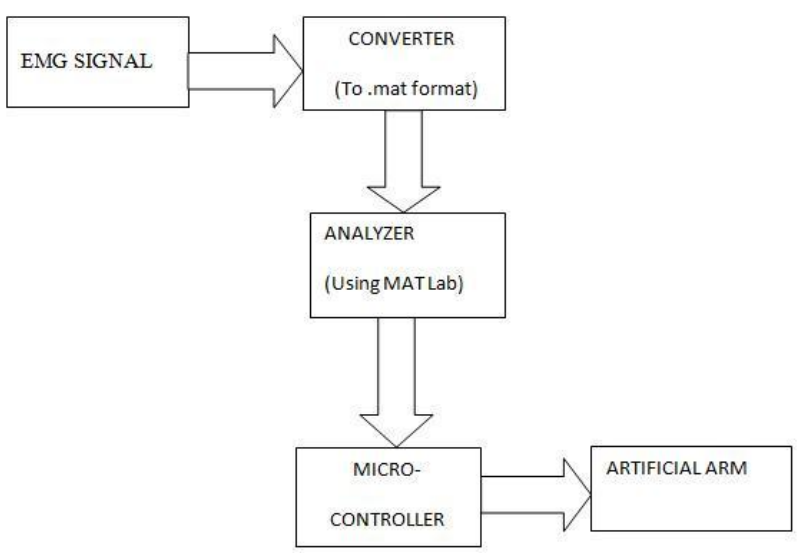

Fig:1 Block Diagram 
The EMG signals obtained from human body are converted to wave format. This '.wav' format is converted to '.txt' forma in MAT Lab. This '.txt' format is analyzed using MAT Lab. It is normalised and rectified. This obtained output is given to microcontroller. The power supply given to the circuit using transformer. The regulator IC is regulated the $12 \mathrm{v}$ to $5 \mathrm{v}$. This $5 \mathrm{v}$ and $12 \mathrm{v}$ are used in circuit. The microcontroller connected to motor which drives the artificial part through relays. Two relays are used to function one motor. Here we are using total 4 relays and two motors.

\subsection{Flow chart}

The relays works as H-bridge with motors.Filtering and rectifications done to get a correct signal for the circuit section. If the maximum peak value of signal is less than 0.17 , then mat lab output gives a value of 3 to controller and the artificial arm moves left. If the maximum peak value of signal is in between 0.17 and 1.11 , then mat lab output gives a value of 1 to controller and the artificial arm picks something. If the maximum peak value of signal is in between 1.11 and 1.2, then mat lab output gives a value of 2 to controller and the artificial arm drops out. If the maximum peak value of signal is greater than 1.2, then mat lab output gives a value of 4 to controller and the artificial arm moves right.

According to the switching of relays motors moves clockwise and anti clockwise. DC motors are the main parts in the circuit section. Based on the rotation of motors, the artificial arm moves. Here the $250 \mathrm{v}$ A/C supply is converted to $12 \mathrm{v}$ AC first using a $250 / 12 \mathrm{v}$ transformer. Then to $12 \mathrm{v}$ DC using a rectifier. This DC voltage is regulated to $5 \mathrm{v}$ for the proper functioning of ICs. Most of the ICs work in $5 \mathrm{v}$. There is a chance to dropout the voltage at the time of rectification. To avoid this we are using capacitors in circuit with appropriate values to minimize them.

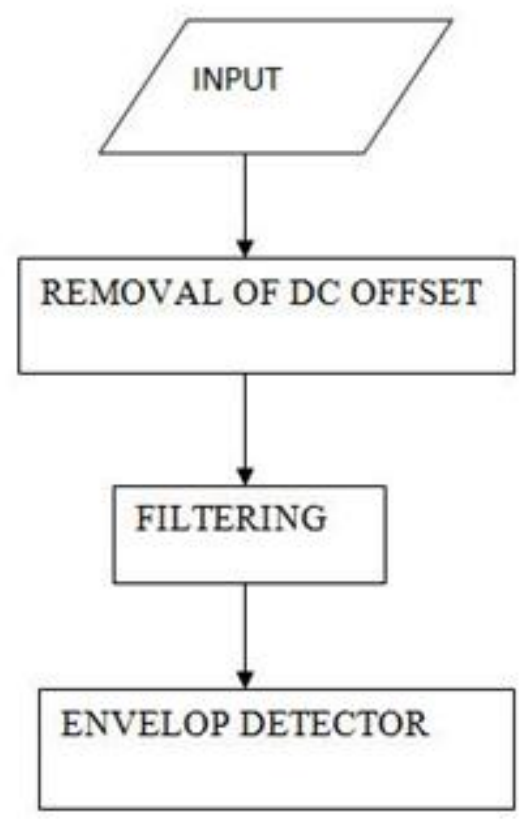

Fig:2 Flow Chart 


\section{RESULT}

Designed and set up the signal processing circuit for "EMG ANALYSIS AND CONTROL OF ARTIFICIAL ARM".Four raw EMG signals took for the functions pick, drop, left movement and right movement. The signals are shown below. We are drawing original signal, rectified signal and the filtered signal here. Signal rectified to take the absolute value. It is easy to work out on peaks from the filtered signal after finding the peaks using function.

\subsection{Drop Out}

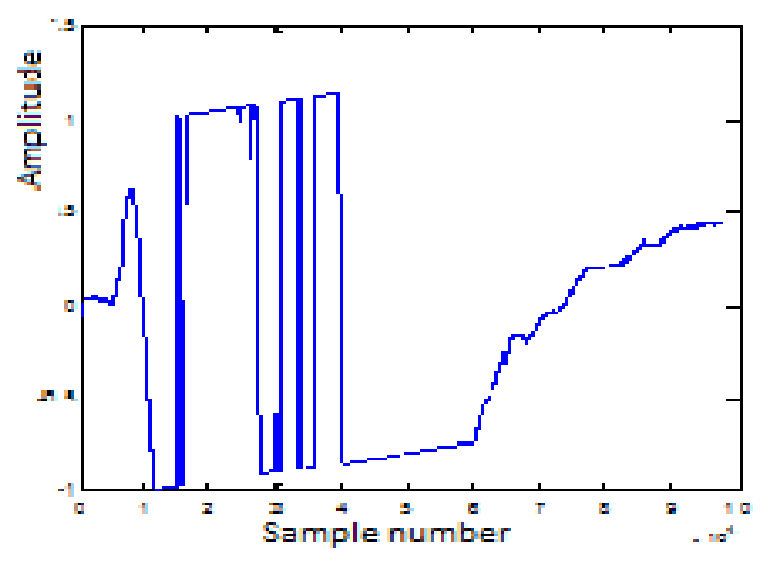

Fig:3 Input Signal for Input

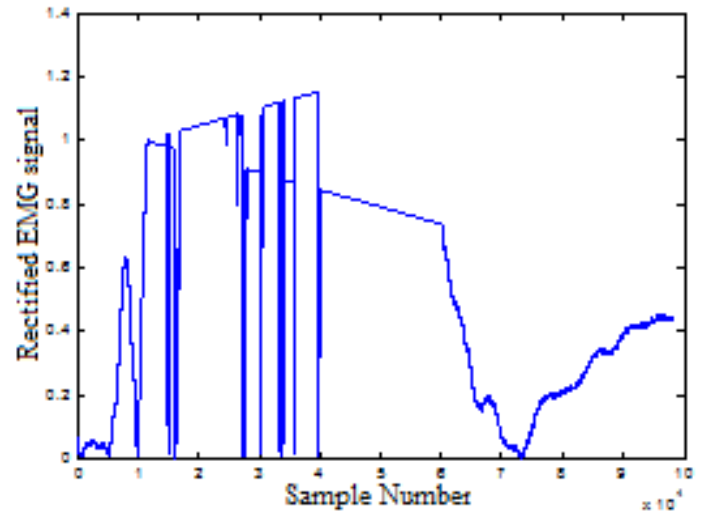

Fig: 4 Rectified Form of Signal 
International Journal on Cybernetics \& Informatics (IJCI) Vol. 5, No. 2, April 2016

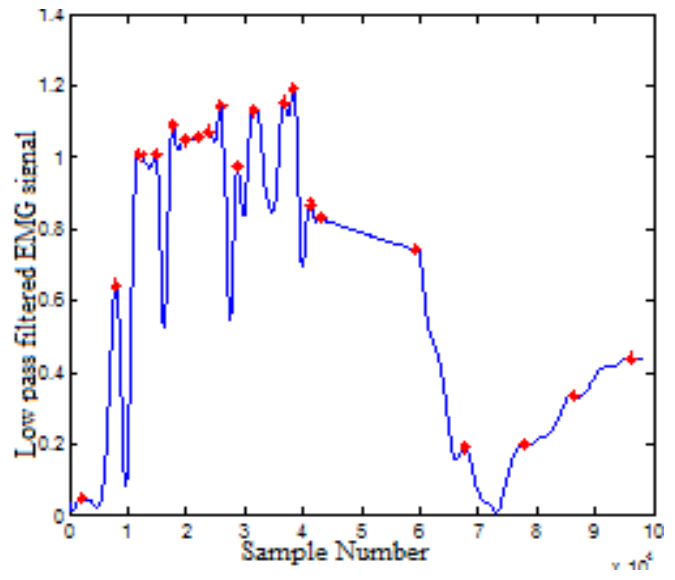

Fig:5 Filtered Form of Signal

\subsection{Pick-up}

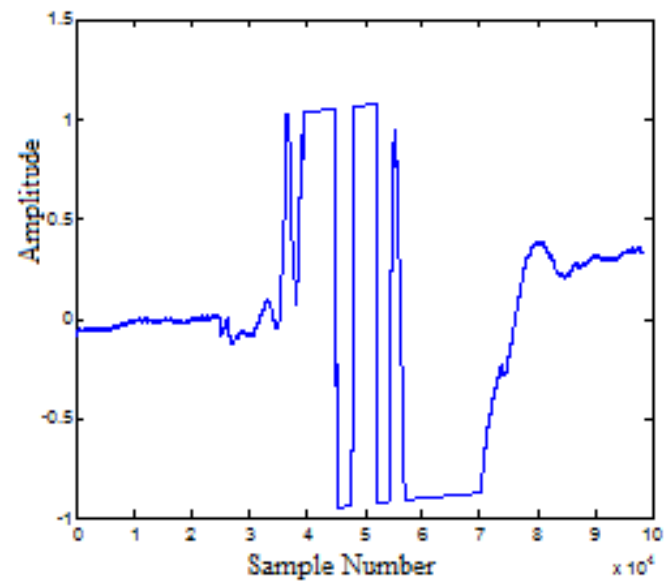

Fig:6 Input Signal To Pick-up

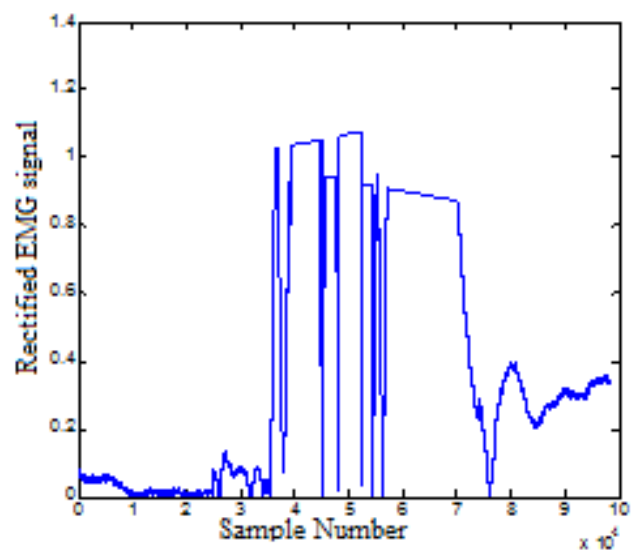

Fig:7 Rectified Form of Signal 
International Journal on Cybernetics \& Informatics (IJCI) Vol. 5, No. 2, April 2016

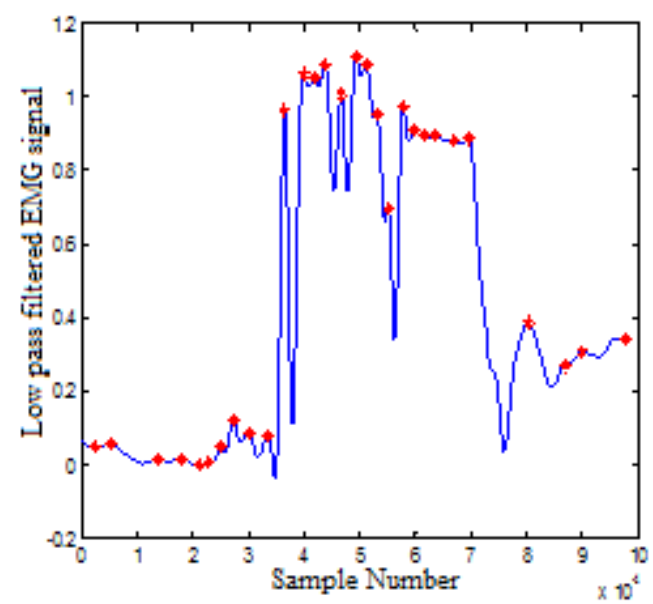

Fig:8 Filtered Form of Signal

\subsection{Left Movement}

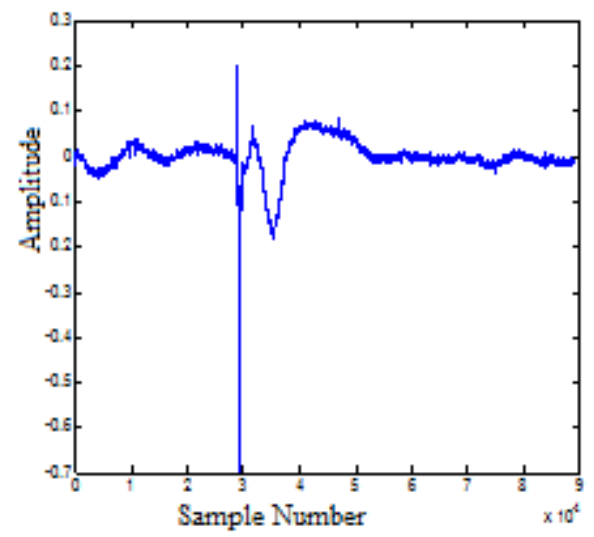

Fig:9 Input Signal For Left Movement

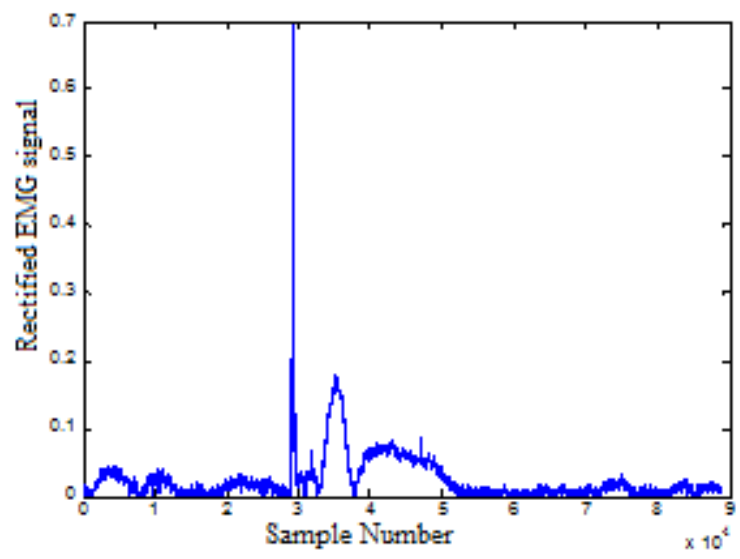

Fig:10 Rectified Form of Signal 
International Journal on Cybernetics \& Informatics (IJCI) Vol. 5, No. 2, April 2016

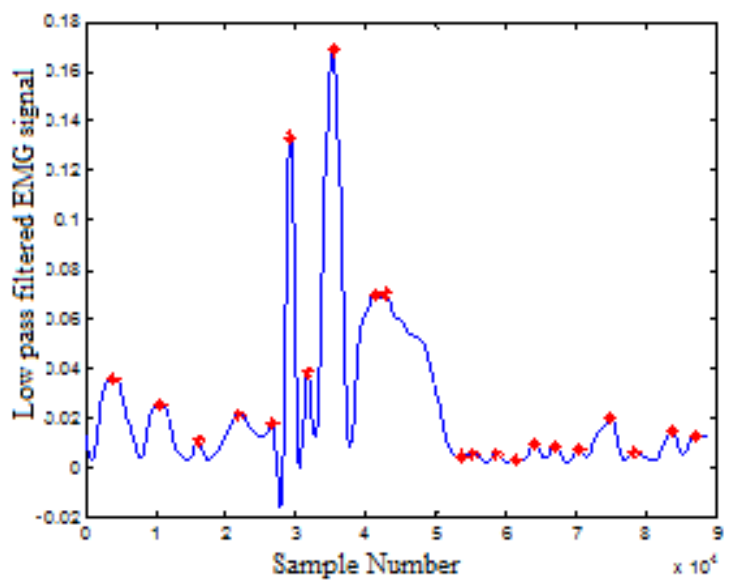

Fig:11 Filtered Form of Signal

\subsection{Right Movement}

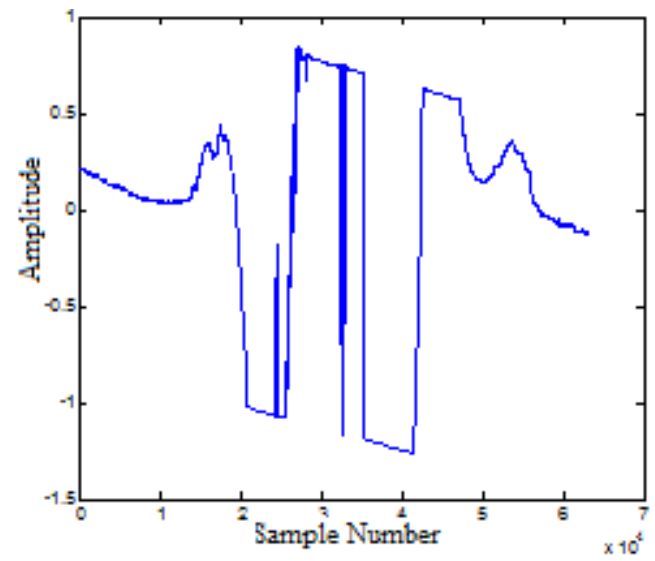

Fig:12 Input Signal For Right Movement

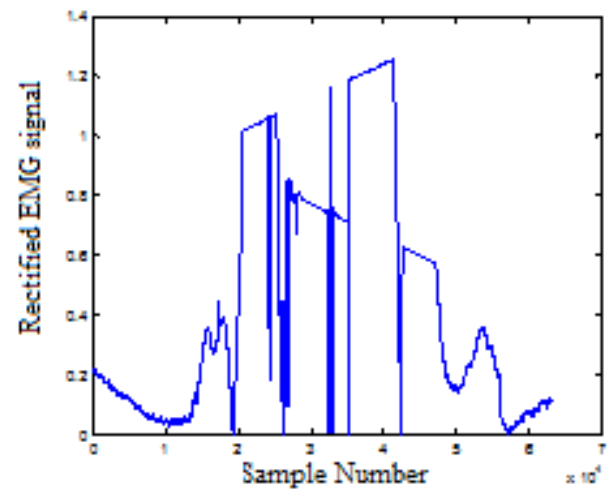

Fig:13 Rectified Form of Signal 


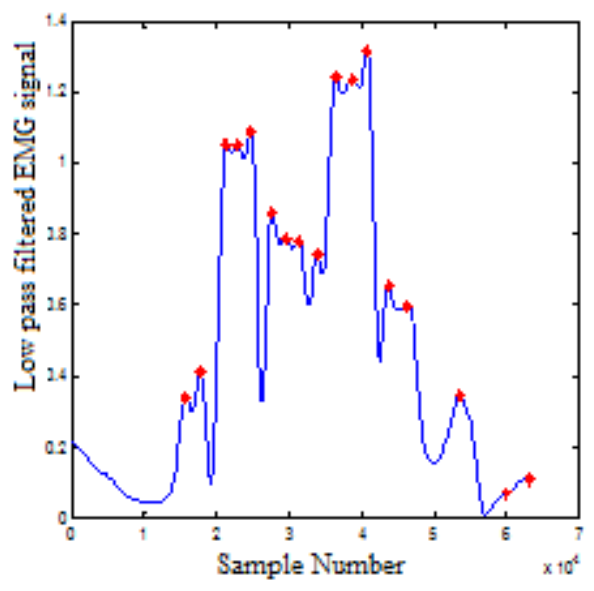

Fig:14 Filtered Form of Signal

Raw EMG signals can be obtained by using a surface electrode placed on arm for various actions such as pick, drop, left movement and right movement. These signals are shown in fig:3,fig:6,fig:9 and fig:12. Then signals are processed. Finally the four filtered signal shown in fig:5,fig:8,fig: 11 and fig:14 are fed to MAT LAB. On the basis of which signal is running in MAT LAB, the artificial arm will be performing above mentioned actions.Fig:13 shows the working model of an artificial arm, which consist of a prototype of an arm.

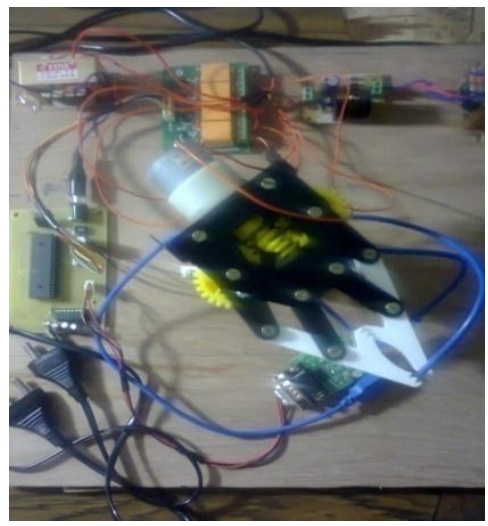

Fig:13 Working Model

\section{CONCLUSION}

EMG can be used to detect muscle fatigue and in treatment of motor disorder problem. Nonetheless, due to insufficient of mathematical model that can fit for general case, more research need to be done for improvement on available model. From the results, it was found that combination of EMG and GA is sufficient for determining best fit model for human lower limb although improvement is still required. The paper has provided an enhanced understanding of human movement in walking motion, which is essential for building of stroke rehabilitation device.This paper presents a novel design and actuation system for a prosthetic hand. The actuation structure was shown to effectively execute the specific actions similar to those found in 
the human hand. Results also validated that the design could be effectively driven by an EMG signal .Complete testing of the actuation system's performance will require the expansion of the EMG inputs to perform different grasps and manipulations.

\section{ACKNOWLEDGEMENT}

The authors would like to thank Abdhu Rahiman ,project coordinator and Dr.Shajee Mohan,Head of the Department of applied electronics and instrumentation of Government Engineering College,Kozhikode for the assistance and support.

\section{REFERENCES}

[1] Dr. Anthony L. Crawford, Jeffrey Molitor, Dr. Alba Perez-Gracia, Dr. Steve C. Chiu, "Design Of A Robotic Hand And Simple EMG Input Controller With A Biologically-Inspired Parallel Actuation System For Prosthetic Applications".

[2] Tan Chee Weil, S.Parasuraman2 And I.Elamvazhuthi ,"Electromyography(EMG) And Human Locomotion"2012 4th International Conference On Computer Engineering And Technology.

[3] Robertoo Merlittie ,Philip A Parker "Electromyography Physiology Engineering And Non-Invasive Application",IEEE,press.

[4] M.C.Carroza,F.Vecci,F.sebastiani,G.Cappiello,S.Roccella,M.Zecca,R.Lazzarini

P.Dairo"Expiremental Analysis Of An Innovative Prosthetic Hand With Proproceptive Sensors" 2003 IEEE International Conference On Robotics And Automation

\section{AUTHORS}

Anjali Raghavan recieved B-Tech degree from University of Calicut in Applied Electronics and Instrumentation.Now pursuing M-Tech degree in A.P.J Abdul Kalam Technological University in VLSI and Embedded System.

Prof. Sunny Joseph is the Head of the Department of Electronics and Communication Engineering in Mar Athanasius College of Engineering, Kothamangalam, Kerala, India. He has a teaching experience of 29 years. He received his B.E from Bangalore University and M.Tech degree from Kerala University. His current research focus is in the area of VHDL, high speed digital design and microwave engineering. He is a member of ISTE and FIE.
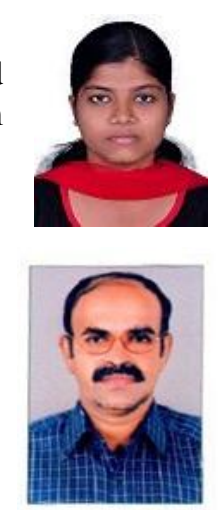